\title{
Analyzing gene expression profiles in dilated cardiomyopathy via bioinformatics methods
}

\author{
Liming Wang ${ }^{1}$, L. Zhu ${ }^{2}$, R. Luan $^{3}$, L. Wang ${ }^{2}$, J. Fu ${ }^{1}$, X. Wang ${ }^{2}$ and L. Sui ${ }^{2}$ \\ ${ }^{1}$ Emergency Department, The Second Affiliated Hospital of Xi'an, Jiaotong University, Xi'an, China \\ ${ }^{2}$ Department of Emergency Medicine, The First Affiliated Hospital of Harbin Medical University, Harbin, China \\ ${ }^{3}$ Medical Department, The First Affiliated Hospital of Harbin Medical University, Harbin, China
}

\begin{abstract}
Dilated cardiomyopathy (DCM) is characterized by ventricular dilatation, and it is a common cause of heart failure and cardiac transplantation. This study aimed to explore potential DCM-related genes and their underlying regulatory mechanism using methods of bioinformatics. The gene expression profiles of GSE3586 were downloaded from Gene Expression Omnibus database, including 15 normal samples and 13 DCM samples. The differentially expressed genes (DEGs) were identified between normal and DCM samples using Limma package in R language. Pathway enrichment analysis of DEGs was then performed. Meanwhile, the potential transcription factors (TFs) and microRNAs (miRNAs) of these DEGs were predicted based on their binding sequences. In addition, DEGs were mapped to the cMap database to find the potential small molecule drugs. A total of 4777 genes were identified as DEGs by comparing gene expression profiles between DCM and control samples. DEGs were significantly enriched in 26 pathways, such as lymphocyte TarBase pathway and androgen receptor signaling pathway. Furthermore, potential TFs (SP1, LEF1, and NFAT) were identified, as well as potential miRNAs (miR-9, miR-200 family, and miR-30 family). Additionally, small molecules like isoflupredone and trihexyphenidyl were found to be potential therapeutic drugs for DCM. The identified DEGs (PRSS12 and FOXG1), potential TFs, as well as potential miRNAs, might be involved in DCM.
\end{abstract}

Key words: Dilated cardiomyopathy; Differentially expressed genes; Pathway enrichment analysis; Transcription factors; microRNAs; Small molecules

\section{Introduction}

Dilated cardiomyopathy (DCM) is characterized by the dilatation of the myocardium, generally associated with systolic dysfunction. DCM, which affects the left ventricle more often than the right, commonly leads to heart failure, and it can result in arrhythmias, thromboembolism and premature death (1). The etiology of DCM is multifactorial, and involves idiopathic, familial, genetic, viral or immune factors $(2,3)$. Nowadays, the molecular mechanism of DCM is still unclear due to its heterogeneity. Therefore, exploring the underlying mechanism of DCM and searching for potential genes involved in DCM are of great significance to human health.

Previous studies revealed that genes, transcription factors (TFs) and microRNAs (miRNAs) are involved in DCM. Mutation in some genes, such as TNNT2, has been suggested to cause DCM (4). The dysregulation of gene expression, like in prospero-related homeobox factor 1 (PROX1), is also involved in DCM. PROX1 can directly repress the expression of fast-twitch skeletal muscle genes (e.g., troponin T3, troponin I2, and myosin light chain 1) at transcriptional level (5). Cardiac-specific knockout of PROX1 causes overexpression of fast-twitch genes, and thus leads to a change from slow-twitch to fast-twitch muscle phenotype, as well as postnatal development of fatal DCM (5). Moreover, GATA4 is one of the cardiac TFs crucial for normal cardiogenesis, and its heterozygous mutations, like p.V39L, p.P226Q and p.T279S, have been found in sporadic DCM patients (6). These mutants can decrease the transcription regulatory activity of GATA4 and reduce the synergistic activation between NKX2-5 and GATA4 (6). In addition, miRNAs like miR-1, miR-29c, miR-30c, miR-30d, miR-149, miR-486, miR-499 are down-regulated in murine phospholamban mutant model of DCM, and the individual silencing of these miRNAs can contribute to cardiac cell loss and heart failure (7).

As a powerful technique, gene expression microarray analysis based on bioinformatics has been widely applied to identify DCM-related genes, possible molecular functions, and biological signal pathways. Based on independent microarray datasets like GSE3585, GSE3586, and GSE1869, a gene expression signature consisting of

Correspondence: X. Wang: <w_xjie@126.com>| L. Sui: <thsuilli@163.com> 
27 genes (e.g., MYH6, MYH10, CCL2, PHLDA1, SNCA, FRZB, SFRP4, SPOCK, CTGF, GOS2, ETV5, and RAR$R E S 1)$ has been identified for DCM, as well as the down-regulation of immune response processes (8). By integrating gene expression profiles with protein-protein interaction (PPI) network analysis, Lin et al. (9) have constructed specific co-expressed PPI networks for DCM and non-DCM samples. Hub proteins in the DCM network tend to be differentially expressed, and two DCM-related functional modules (muscle contraction and organ morphogenesis) have also been identified (9). Using the microarrays of GSE3586, Xiao et al. (10) identified the functional modules related to heart failure with different etiologies. However, these studies focused mainly on genes, and little is said about the TFs or miRNAs that regulate gene expression, or about the potential molecular drugs for DCM treatment.

In this study, we re-analyzed the gene expression profile of GSE3586 to explore the molecular mechanism of DCM at both the gene expression level and expression regulation level. Differentially expressed genes (DEGs) were identified between DCM and normal samples. DEGs bio-functions, potential TFs, and potential miRNAs, as well as small molecules that could be employed in the treatment of DCM, were also investigated.

\section{Material and Methods}

\section{Microarray data}

The gene expression profile of GSE3586 (8) was obtained from the Gene Expression Omnibus (GEO) database (http://www.ncbi.nlm.nih.gov/geo/) based on the platform of Human Unigene 3.1 cDNA Array 37.5K v1.0. A total of 28 samples were available, including $13 \mathrm{DCM}$ samples from septal myocardial tissue of DCM patients and 15 normal samples from non-failing donor hearts of healthy controls.

\section{Data preprocessing and DEGs screening}

As the intrinsic background of different chips might affect the calculation of expression values, the raw data of each chip were first normalized using the Geoquery package (version 2.34.0, available at http://www.bioconductor. org/packages/release/bioc/html/GEOquery.html) (11) in $\mathrm{R}$ language. After, the expression data were log2 transformed, and a linear regression model was constructed to compare the gene expression in DCM and control samples. Then, the Benjamini and Hochberg $(\mathrm{BH})$ procedure (12) was applied to adjust $P$ values and thus obtain false discovery rate (FDR). The Limma package (13) in R language was used to identify the DEGs in DCM and control samples. Finally, the log2 fold change $(F C) \neq 0$ and FDR $<0.05$ were chosen as the cutoff values.

\section{Hierarchical clustering analysis}

For the identified DEGs, hierarchical clustering analysis was performed by using the pheatmap package (version 1.0.2, available at http://cran.r-project.org/web/ packages/pheatmap/index.html) created by $\mathrm{R}$ Core Team (Austria).

\section{Pathway analysis of DEGs}

All the metabolic and non-metabolic pathways that involved DEGs were analyzed by using the Gene Set Analysis Toolkit V2 (WebGestalt2) platform (Vanderbilt University, Nashville, TN, USA; available at http://bioinfo. vanderbilt.edu/webgestalt) (14) based on the Wikipathways database (15). $P$ values less than 0.05 and the number of involved genes $\geqslant 2$ were selected as cut-off criteria.

\section{Potential TFs and miRNAs}

TFs and miRNAs play crucial roles in DCM. The Molecular Signature Database version 3.0 (MSigDB 3.0, available at http://www.broadinstitute.org/msigdb) (16) contains hallmark, positional, curated, motif, and computational gene sets, gene ontology, oncogenic signatures, and immunologic signatures. Among these, motif gene sets include genes that share a conserved and cataloged cisregulatory motif in promoters and in $3^{\prime}$-untranslated regions (3'-UTRs). Specifically, motif gene sets contain TF targets that share a TF binding site recorded in the TRANSFAC database (version 7.4, http://www.gene-regulation.com/), as well as miRNA targets that share a $3^{\prime}$-UTR miRNA binding motif. In this study, based on the well-annotated motif gene sets in MSigDB 3.0, Gene Set Enrichment analysis (GSEA) (17) was performed to identify the potential TFs and miRNAs of DEGs by utilizing hypergeometric distribution. The criterion was set as FDR <0.05.

\section{Identification of potential small molecules associated with DCM}

Currently, the connectivity map (cMap) database includes 7056 gene-expression datasets that involve 6100 small molecule treatment-control pairs (18). In order to identify the small molecules that could simulate the normal or DCM cells, the identified DEGs (up- and downregulated genes) were mapped to the small molecules deposited in cMap database using GSEA (17). The correlation score was calculated, ranging from -1 to 1 .

\section{Results}

\section{Screening, hierarchical clustering, and pathway analysis of DEGs}

After data normalization (Figure $1 \mathrm{~A}$ and $\mathrm{B}$ ) and differential expression analysis, a total of 4777 DEGs were identified in DCM and normal samples, including 2711 up-regulated genes and 2066 down-regulated genes. Genes like PRSS12 (protease serine 12, log2 FC= -0.167 and FDR=0.003) and FOXG1 (forkhead box G1, $\log 2 \mathrm{FC}=-0.156$ and FDR=0.045) were significantly downregulated in DCM samples in comparison with normal samples. In addition, the expressions of 13 DEGs were 
A
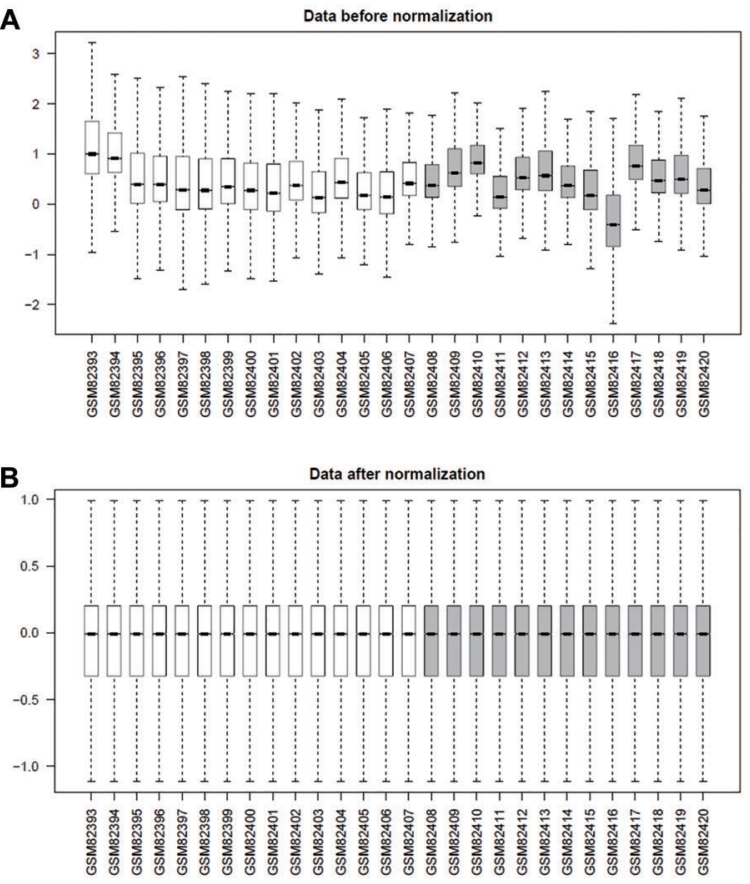

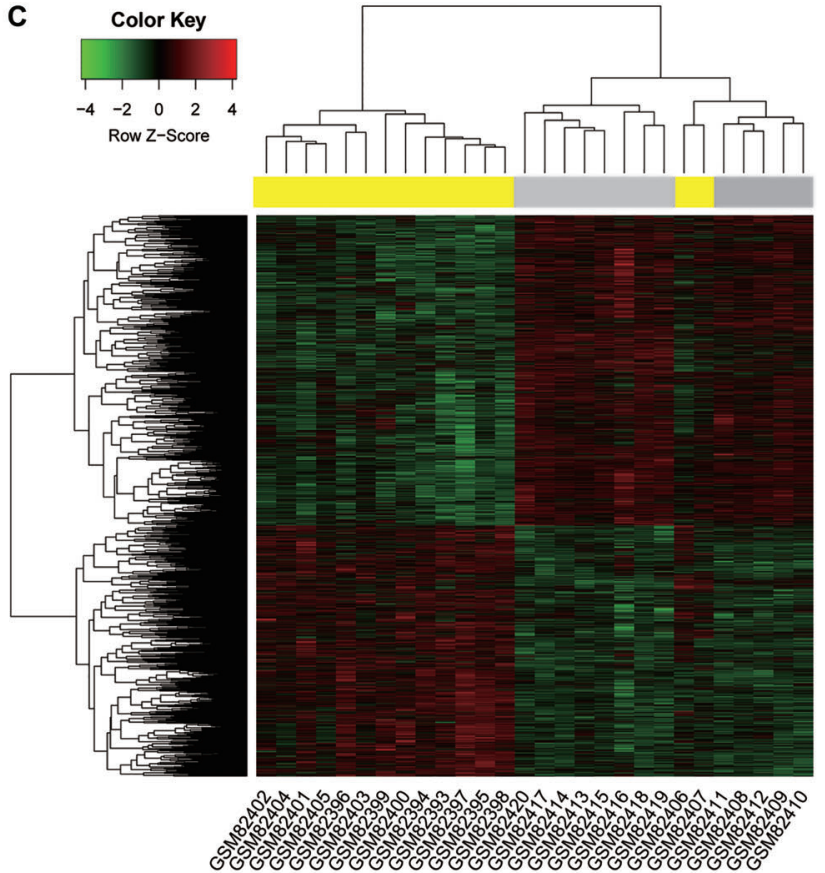

Figure 1. Normalization of gene expression data and hierarchical clustering of differentially expressed genes (DEGs). $A$, gene expression profiles before normalization. White boxes represent normal samples, while gray boxes stand for dilated cardiomyopathy samples. $B$, gene expression profiles after normalization. $C$, hierarchical clustering of DEGs. Yellow boxes represent normal samples, while gray boxes stand for dilated cardiomyopathy samples. The green and red bars represent low and high expression levels, respectively. GSMxxxxx: the accession number of a certain sample in the Gene Expression Omnibus database.

elevated more than 2-fold, while only 1 gene (CCL2) was down-regulated more than 2-fold. For the identified DEGs, hierarchical clustering analysis was performed, and samples were classified into two clusters (Figure 1C). Surprisingly, 2 normal samples were clustered with DCM samples rather than with normal samples. This might be caused by intrinsic deviation of the clustering tool (namely, the pheatmap package), potential sub-healthy state of control individuals, and experimental deviation. In general, samples in normal and DCM groups could be distinguished based on DEGs expression. After pathway analysis based on Wikipathways database $(15,19)$, a total of 26 significant pathways were identified (Table 1), among which 8 pathways were associated with signaling and 2 pathways were involved in cancer. A total of 124 DEGs (e.g., POLA2, TMED7, SLC25A24, NF2, and COL4A2) were significantly enriched in "lymphocyte TarBase pathway" (P value $\left.=9.35 \times 10^{-16}\right)$, while 28 DEGs (e.g., NCOR2, DSTN, RAC1, PIK3R1, and FHL2) were significantly enriched in "androgen receptor signaling pathway" $\left(P\right.$ value $\left.=9.12 \times 10^{-7}\right)$.

\section{Potential TFs and miRNAs}

The top 10 target sites and corresponding TFs with a highly significant correlation are listed in Table 2, and SP1, lymphocyte enhancer factor-1 (LEF1), and nuclear factor of activated T cells (NFAT) were among the most significant TFs. SP1 and LEF1 collectively targeted 191 genes; LEF1 and NFAT collectively targeted 149 genes, like down-regulated PRSS12; SP1 and NFAT collectively targeted 147 genes; SP1, LEF1, and NFAT collectively targeted 58 genes.

In addition, the potential miRNAs of DEGs were screened, and miR-9, miR-200 family, and miR-30 family were among the most significant miRNAs (Table 2). More specifically, miR-9, miR-200 family, and miR-30 family collectively targeted the down-regulated FOXG1.

\section{Potential small molecules associated with DCM}

Based on the cMap database, we performed GSEA for DEGs to screen for possible small molecule drugs. Finally, 20 small molecules were identified as having a highly significant correlation with DCM (Table 3), including 6 negatively correlated and 14 positively correlated small molecules. Among these molecules, isoflupredone and trihexyphenidyl could be potential small molecule drugs for DCM treatment, and DL-thiorphan and milrinone might trigger DCM (Table 3).

\section{Discussion}

DCM is characterized by ventricular dilatation, and it commonly leads to heart failure. Although many studies 
Table 1. Biological pathways enriched by differentially expressed genes (DEGs) in dilated cardiomyopathy.

\begin{tabular}{|c|c|c|c|}
\hline Pathway & $\mathrm{P}$ value & Gene count & Genes \\
\hline Muscle cell TarBase & $7.25 \mathrm{E}-16$ & 107 & POLA2, TMED7, SLC25A24, NF2, LUZP1, et al. \\
\hline Lymphocyte TarBase & 9.35E-16 & 124 & POLA2, TMED7, SLC25A24, NF2, COL4A2, et al. \\
\hline Insulin signaling & $3.14 \mathrm{E}-13$ & 54 & MAPK8, MAP2K5, MINK1, RAF1, RAC1, et al. \\
\hline Epithelium TarBase & $5.61 \mathrm{E}-11$ & 81 & TMED7, SLC25A24, COL4A2, CUL4B, STX7, et al. \\
\hline G protein signaling pathways & 7.13E-11 & 36 & AKAP9, GNG12, AKAP5, GNG3, PDE4B, et al. \\
\hline MAPK signaling pathway & $2.77 \mathrm{E}-10$ & 49 & MAPK8, TRAF6, MAP2K5, BRAF, MINK1, et al. \\
\hline EGF-EGFR signaling pathway & 1.24E-09 & 49 & MAPK8, MAP2K5, BRAF, SH3GL3, RAF1, et al. \\
\hline Integrated pancreatic cancer pathway & 2.32E-09 & 50 & MAPK8, CDKN2B, LTBP1, LEFTY1, RAF1, et al. \\
\hline TSH signaling pathway & $2.54 \mathrm{E}-09$ & 28 & PDPK1, BRAF, RAF1, CDK4, PIK3R1, et al. \\
\hline Myometrial relaxation and contraction pathways & $3.79 \mathrm{E}-09$ & 46 & RGS16, GNG12, RYR1, GNG3, PDE4B, et al. \\
\hline Translation factors & $5.32 \mathrm{E}-09$ & 23 & EIF3C, EIF4B, EIF3D, EIF1AX, EIF4A2, et al. \\
\hline Calcium regulation in the cardiac cell & 1.10E-08 & 43 & RGS16, GNG12, RYR1, GNG3, CAMK1, et al. \\
\hline Focal adhesion & 3.33E-08 & 48 & MAPK8, MAP2K5, BRAF, COL4A2, PAK7, et al. \\
\hline Signaling pathways in glioblastoma & 4.53E-08 & 29 & PDPK1, CDKN2B, MAP2K5, BRAF, MAP2K7, et al. \\
\hline Squamous cell TarBase & $8.19 E-08$ & 41 & HOXA7, TMED7, NF2, CUL4B, PPP1R7, et al. \\
\hline Regulation of actin cytoskeleton & $1.01 \mathrm{E}-07$ & 42 & GNG12, SSH2, BRAF, FGF13, PAK7, et al. \\
\hline Adipogenesis & 1.13E-07 & 37 & NCOR2, EPAS1, MEF2A, CDKN1A, RETN, et al. \\
\hline Electron transport chain & 1.13E-07 & 32 & ATP5L, NDUFA5, NDUFV2, ATPIF1, NDUFC1, et al. \\
\hline mRNA processing & 1.68E-07 & 37 & HNRNPA1, SNRPA1, CELF2, SRRM1, DHX9, et al. \\
\hline Diurnally regulated genes with circadian orthologs & $2.15 \mathrm{E}-07$ & 20 & UGP2, HSPA8, PPP2CB, DAZAP2, EIF4G2, et al. \\
\hline Integrin-mediated cell adhesion & $2.15 \mathrm{E}-07$ & 31 & PDPK1, VAV3, MAP2K5, PXN, BRAF, et al. \\
\hline Androgen receptor signaling pathway & $9.12 \mathrm{E}-07$ & 28 & NCOR2, DSTN, RAC1, PIK3R1, FHL2, et al. \\
\hline TGF beta signaling pathway & 1.14E-06 & 38 & MAPK8, TRAF6, RAF1, RAC1, MAP2K1, et al. \\
\hline miRs in muscle cell differentiation & 2.69E-06 & 14 & PRKCE, PRKD3, PRKCB, PRKAR1B, PRKCZ, et al. \\
\hline Proteasome degradation & 2.93E-06 & 22 & PSMA3, UBA1, PSMA2, PSME2, PSMB7, et al. \\
\hline Integrated breast cancer pathway & $6.81 \mathrm{E}-06$ & 22 & MYCBP2, CDC25A, PHB, CERK, VEGFA, et al. \\
\hline
\end{tabular}

have been devoted to exploring the pathogenesis of DCM, the mechanism of DCM development and progression still remains largely unknown. In the present study, we identified the DEGs between DCM and normal samples, performed pathway analysis for DEGs, and predicted potential TFs, miRNAs, and small molecules related with DCM.

Bioinformatics gene expression microarray analysis has been widely used to identify DCM-related genes. Based on the microarrays of GSE3586, Barth et al. (8) found 1353 up-regulated transcripts and 384 downregulated transcripts by using 2-class unpaired significance analysis of microarrays with the criteria of FDR $<0.05$ and fold change $\geqslant 1.2$. However, we screened out 4777 DEGs by utilizing Limma package with the criteria of FDR $<0.05$ and $\log 2 \mathrm{FC} \neq 0$. The differences in $\mathrm{DEGs}$ number between our study and the previous study might be caused by the different analysis methods and criteria; however, our method and criteria are the most commonly used in DEG screening.

Moreover, the previous study has proven that the immune response process is involved in end-stage DCM (8), and we identified that the lymphocyte TarBase pathway and androgen receptor signaling pathway were significantly enriched by DEGs between DCM and normal samples. Reportedly, the lymphocyte is an important part of the immune system and has a vital role in heart function (20). Neutrophil/lymphocyte ratio is associated with the low function capacity in DCM patients (21). In androgen receptor signaling pathway, the androgen receptor is involved in the inflammation response and affects myocardial function directly (22). Thus, we suggested that lymphocyte TarBase pathway and androgen receptor signaling pathway might play roles in DCM via immune system.

Generally, TFs can play pivotal roles in regulating the transcription process of encoding genes. In this study, we also predicted potential TFs of DEGs, such as LEF1, NFAT, and SP1. Being expressed in pre-B and T lymphocytes, LEF1 is a regulatory participant in lymphocyte gene expression and differentiation (23). The transcriptional activity of LEF1 is related to heart function via plakoglobin (24). Besides, the translocation of $\beta$-cateninTCF/LEF-1 complex into the nucleus is involved in Wnt/ wingless signal transduction pathway activated by ATP depletion to modulate the expression of genes, which can regulate cell proliferation, apoptosis, and differentiation $(25,26)$. Thus, we speculated that $L E F 1$ might be related 
Table 2. Top 10 potential transcription factors and miRNAs of differentially expressed genes (DEGS) in dilated cardiomyopathy.

\begin{tabular}{|c|c|c|c|}
\hline Target sequence & Regulator & $P$ value & Target genes \\
\hline \multicolumn{4}{|l|}{ Transcription factor } \\
\hline hsa_GGGCGGR & SP1 & $9.24 \mathrm{E}-169$ & ARHGAP26, TRIM24, RYR1, PRSS12, RAB2A, et al. \\
\hline hsa_AACTTT & Unknown & $1.33 \mathrm{E}-134$ & PRSS12, PPP2CB, CACNA1H, CTBP2, MORF4L2, et al. \\
\hline hsa_TTGTTT & FOXO4 & $3.92 \mathrm{E}-117$ & ASPA, HAS2, MIS12, PCDH7, FBXO32, et al. \\
\hline hsa_CTTTGT & LEF1 & $2.71 \mathrm{E}-112$ & GBE1, TRIM24, MORF4L2, PPP1R16B, MYH10, et al. \\
\hline hsa_CAGGTG & $E 12$ & 4.39E-97 & ARHGAP26, RYR1, CCNYL1, PRSS12, TMEM26, et al. \\
\hline hsa_GGGAGGRR & $M A Z$ & 4.39E-97 & ZBTB10, RYR1, PRSS12, FGD2, RAB2A, et al. \\
\hline hsa_TGGAAA & NFAT & 2.97E-96 & ZBTB10, ASPA, PRSS12, FAM120C, HIST2H2BE, et al. \\
\hline hsa_SCGGAAGY & ELK1 & $4.89 \mathrm{E}-68$ & MDM4, PTPN23, RAB2A, DPP8, COMMD6, et al. \\
\hline hsa_CAGCTG & AP4 & $5.70 \mathrm{E}-63$ & ACVR1, RYR1, ASPA, TSC22D3, SPIN2A, et al. \\
\hline hsa_CACGTG & MYC & $5.43 \mathrm{E}-57$ & ZBTB10, PPCS, CCNYL1, SPIN2A, RAB2A, et al. \\
\hline \multicolumn{4}{|r|}{ ( } \\
\hline has_TTTGCAC & miR-19a, miR-19b & $3.24 \mathrm{E}-46$ & UBL3, ZBTB10, PLXNC1, INO80, DLC1, et al. \\
\hline hsa_CAGTATT & miR-200b, miR-200c, miR-429 & $3.30 \mathrm{E}-46$ & PHACTR3, CCNYL1, NPM1, FOXG1, YPEL2, et al. \\
\hline hsa_TGTTTAC & $\begin{array}{l}\text { miR-30a-5p, miR-30c, miR-30d, } \\
\text { miR-30b, miR-30e-5p }\end{array}$ & $1.35 \mathrm{E}-44$ & ZDHHC17, ACVR1, UBN1, FOXG1, YPEL2, et al. \\
\hline hsa_ACCAAAG & miR-9 & $1.23 \mathrm{E}-42$ & LRRTM4, FAF2, CUL4A, FOXG1, YPEL2, et al. \\
\hline hsa_GTGCCTT & miR-506 & $3.97 \mathrm{E}-42$ & FAF2, FAM53B, TMEM184B, RYR1, UBN1, et al. \\
\hline hsa_TGAATGT & $\begin{array}{l}\text { miR-181a, miR-181b, miR-181c, } \\
\text { miR-181d }\end{array}$ & $2.13 \mathrm{E}-41$ & HECA, ACSL1, INO80, EPHA4, PNRC2, et al. \\
\hline hsa_TTGCACT & miR-130a, miR-301, miR-130b & $2.68 \mathrm{E}-41$ & HECA, ACVR1, FAM53B, ACSL1, INO80, et al. \\
\hline hsa_TGCTGCT & $\begin{array}{c}\text { miR-15a, miR-16, miR-15b, miR-195, } \\
\text { miR-424, miR-497 }\end{array}$ & $4.00 \mathrm{E}-40$ & ZBTB10, ACSL1, LUZP1, TSC22D3, VAMP8, et al. \\
\hline hsa_GCACTTT & $\begin{array}{c}\text { miR-17-5p, miR-20a, miR-106a, } \\
\text { miR-106b, miR-20b, miR-519d }\end{array}$ & $3.46 \mathrm{E}-39$ & HECA, LUZP1, EPAS1, INO80, EPHA4, et al. \\
\hline hsa_TGCTTTG & miR-330 & 3.37E-38 & DIP2B, ACVR1, MAP2K5, LUZP1, NF2, et al. \\
\hline
\end{tabular}

miRNAs: microRNAs.

with DCM. In addition, NFAT is expressed in immunesystem cells and plays a vital role in the transcription of cytokine genes and other genes which were critical for the immune response (27). NFAT is also a critical regulator of cardiac development and myocyte maturation (28), and the translocation of dephosphorylated NFAT-3 to nucleus in DCM can activate the calcineurin signaling pathway (29), whose activation can induce atrial hypertrophy during atrial fibrillation (30). Specifically, LEF1 and NFAT collectively targeted the down-regulated PRSS12, which is also named neurotrypsin or motopsin. Reportedly, neurotrypsin can produce C-terminal agrin fragment that plays crucial roles in the initiation and maintenance of neuromuscular junctions and is a biomarker of muscle wasting in congestive heart failure patients (31). This evidence indicated that these TFs might play roles in $\mathrm{DCM}$. Also, SP1 can regulate the expression of $\mathrm{NF}-\kappa \mathrm{B}$ related cytokines, and is associated with inflammation in aortic aneurysm and heart failure (32). Also, SP1 participates in androgen receptor signaling pathway. Although there is very few evidence showing that $S P 1$ is involved in DCM, we speculated that SP1 might associate with DCM, as SP1 and NFAT collectively targeted 147 genes.
miRNAs can post-transcriptionally regulate gene expression, and act as pivotal modulators in DCM (7). In this research, potential miRNAs of DEGs were identified, and miR-9 was the most significant one. miR-9 can regulate NFATc3-mediated hypertrophy signaling (33), and NFAT was identified in our study. Gladka et al. (34) reported that miR-9 was involved in dilated cardiac hypertrophy on a regulatory perspective, and miRNAs in miR-200 family are related with DCM and heart failure (35). miR-200a can regulate $\beta$-catenin expression and subsequently modulate Wnt/ $\beta$-catenin signaling (36), while there is little information that focuses on the function of miR-200b or miR-200c in DCM. Besides, miR-30c and miR-30d are down-regulated in murine model of DCM, and their silencing contributes to cardiac cell loss and heart failure (7). Specifically, miR-9, miR-200 family, and miR-30 family collectively targeted the down-regulated FOXG1, which belongs to the forkhead box family, and the deletion of Foxm1 leads to diminished DNA replication and mitosis in cardiomyocytes, and ventricular hypoplasia in Foxm $1^{-/-}$mouse line (37). Therefore, we speculate that miR-9, miR-200 family, and miR-30 family might play vital roles in DCM. 
Table 3. Enriched significant small molecules.

\begin{tabular}{lcc}
\hline Molecule in cMap & Enrichment score & P value \\
\hline Isoflupredone & -0.928 & 0.00054 \\
Trihexyphenidyl & -0.922 & 0.00080 \\
Prestwick-691 & -0.856 & 0.00599 \\
Ajmaline & -0.834 & 0.00903 \\
Carteolol & -0.754 & 0.00734 \\
Thiamphenicol & -0.672 & 0.00883 \\
DL-thiorphan & 0.942 & 0.00632 \\
Milrinone & 0.903 & 0.00182 \\
Apigenin & 0.873 & 0.00036 \\
Ronidazole & 0.852 & 0.00611 \\
Skimmianine & 0.835 & 0.00115 \\
Chrysin & 0.826 & 0.01064 \\
Rottlerin & 0.825 & 0.01074 \\
Clomipramine & 0.787 & 0.00400 \\
Deptropine & 0.783 & 0.00412 \\
Sulconazole & 0.783 & 0.00416 \\
Ethaverine & 0.741 & 0.00863 \\
Ketanserin & 0.727 & 0.01110 \\
Prestwick-685 & 0.670 & 0.01045 \\
Resveratrol & 0.536 & 0.00558 \\
\hline
\end{tabular}

Furthermore, the expression patterns of DEGs between normal and DCM samples were mapped to cMap database, and a series of small molecules were predicted to correlate with DCM. Small molecules isoflupredone and trihexyphenidyl were found to be potential drugs for the prevention and treatment of DCM. Isoflupredone is mainly used in inflammatory diseases, and it is effective in the endotoxin-induced mastitis and can significantly improve

\section{References}

1. Fatkin D, Otway R, Richmond Z. Genetics of dilated cardiomyopathy. Heart Fail Clin 2010; 6: 129-140, doi: 10.1016/ j.hfc.2009.11.003.

2. Caforio AL, Bottaro $\mathrm{S}$, lliceto $\mathrm{S}$. Dilated cardiomyopathy (DCM) and myocarditis: Classification, clinical and autoimmune features. Appl Cardiopulm Pathophysiol 2012; 16: 82-95.

3. McNally EM, Golbus JR, Puckelwartz MJ. Genetic mutations and mechanisms in dilated cardiomyopathy. $J$ Clin Invest 2013; 123: 19-26, doi: 10.1172/JCl62862.

4. Campbell N, Sinagra G, Jones KL, Slavov D, Gowan K, Merlo $M$, et al. Whole exome sequencing identifies a troponin $\mathrm{T}$ mutation hot spot in familial dilated cardiomyopathy. PLoS One 2013; 8: e78104, doi: 10.1371/journal. pone.0078104.

5. Petchey L, Risebro C, Vieira JM, Roberts T, Bryson J, Greensmith L, et al. Loss of Prox 1 in striated muscle causes slow to fast skeletal muscle fiber conversion and dilated cardiomyopathy. Proc Natl Acad Sci U S A 2014; 111: 9515-9520, doi: 10.1073/pnas.1406191111. lung function in inflammatory airway disease $(38,39)$. Additionally, trihexyphenidyl is considered a treatment option for idiopathic cervical dystonia (40), while it is unknown whether trihexyphenidyl has effects on DCM. Therefore, it needs to be further researched whether these two small molecules are useful in treating DCM.

In conclusion, our study identified DEGs between DCM and control samples (e.g., PRSS12 and FOXG1), and these DEGs participated in significant pathways such as lymphocyte TarBase pathway and androgen receptor signaling pathway. Potential TFs (LEF1, SP1 and NFAT) and miRNAs (miR-9, miR-200 family, and miR-30 family) might play roles in DCM. Furthermore, two small molecules (isoflupredone and trihexyphenidyl) might be capable of treating DCM. This result might provide new insight into understanding the molecular mechanism of DCM and finding new therapeutic targets of DCM.

More experiments are needed to verify these results, as they were obtained through bioinformatics analysis. We are planning to perform gene overexpression and silencing analysis to investigate the roles of potential DCM-related genes, transcription factors, and miRNAs in DCM. Moreover, we are also planning to use animal models to study the therapeutic effects of the potential drugs isoflupredone and trihexyphenidyl, identified in this research.

\section{Acknowledgements}

This study was supported by Heilongjiang Provincial Health Department of Scientific Research Subject (2012565) and Heilongjiang Provincial Department of Education Foundation of China (11521151)
6. Li J, Liu W, Yang Z, Yuan F, Xu L, Li R, et al. Prevalence and spectrum of GATA4 mutations associated with sporadic dilated cardiomyopathy. Gene 2014; 548: 174-181.

7. Isserlin R, Merico D, Wang D, Vuckovic D, Bousette N, Gramolini AO, et al. Systems analysis reveals downregulation of a network of pro-survival miRNAs drives the apoptotic response in dilated cardiomyopathy. Mol Biosyst 2015; 11: 239-251, doi: 10.1039/C4MB00265B.

8. Barth AS, Kuner R, Buness A, Ruschhaupt M, Merk S, Zwermann L, et al. Identification of a common gene expression signature in dilated cardiomyopathy across independent microarray studies. J Am Coll Cardiol 2006; 48: 1610-1617, doi: 10.1016/j.jacc.2006.07.026.

9. Lin CC, Hsiang JT, Wu CY, Oyang YJ, Juan HF, Huang HC. Dynamic functional modules in co-expressed protein interaction networks of dilated cardiomyopathy. BMC Syst Biol 2010; 4: 138, doi: 10.1186/1752-05094-138.

10. Xiao Y, Xu C, Xu L, Guan J, Ping Y, Fan H, et al. Systematic identification of common functional modules related to heart 
failure with different etiologies. Gene 2012; 499: 332-338, doi: 10.1016/j.gene.2012.03.039.

11. Davis S, Meltzer PS. GEOquery: a bridge between the Gene Expression Omnibus (GEO) and BioConductor. Bioinformatics 2007; 23: 1846-1847, doi: 10.1093/bioinformatics/ btm254.

12. Benjamini $Y$, Yekutieli D. The control of the false discovery rate in multiple testing under dependency. Annals of Statistics 2001; 29: 1165, doi: 10.1214/aos/1013699998.

13. Smyth GK. Limma: linear models for microarray data. In: Anonymous, Bioinformatics and computational biology solutions using $R$ and Bioconductor. New York: Springer; 200. p 397-420.

14. Duncan D, Prodduturi N, Zhang B. WebGestalt2: an updated and expanded version of the Web-based Gene Set Analysis Toolkit. BMC Bioinformatics 2010; 11: P10, doi: 10.1186/1471-2105-11-S4-P10.

15. Kelder T, van lersel MP, Hanspers K, Kutmon M, Conklin $\mathrm{BR}$, Evelo CT, et al. WikiPathways: building research communities on biological pathways. Nucleic Acids Res 2012; 40: D1301-D1307, doi: 10.1093/nar/gkr1074.

16. Liberzon A, Subramanian A, Pinchback R, Thorvaldsdottir $\mathrm{H}$, Tamayo P, Mesirov JP. Molecular signatures database (MSigDB) 3.0. Bioinformatics 2011; 27: 1739-1740, doi: 10.1093/bioinformatics/btr260.

17. Subramanian A, Tamayo P, Mootha VK, Mukherjee S, Ebert BL, Gillette MA, et al. Gene set enrichment analysis: a knowledge-based approach for interpreting genome-wide expression profiles. Proc Natl Acad Sci U S A 2005; 102: 15545-15550, doi: 10.1073/pnas.0506580102.

18. Lamb J, Crawford ED, Peck D, Modell JW, Blat IC, Wrobel MJ, et al. The Connectivity Map: using gene-expression signatures to connect small molecules, genes, and disease. Science 2006; 313: 1929-1935, doi: 10.1126/science. 1132939.

19. Pico AR, Kelder T, van lersel MP, Hanspers $K$, Conklin BR, Evelo C. WikiPathways: pathway editing for the people. PLoS Biol 2008; 6: e184, doi: 10.1371/journal.pbio.0060184.

20. Maisel AS, Knowlton KU, Fowler P, Rearden A, Ziegler MG, Motulsky $\mathrm{HJ}$, et al. Adrenergic control of circulating lymphocyte subpopulations. Effects of congestive heart failure, dynamic exercise, and terbutaline treatment. $J$ Clin Invest 1990; 85: 462-467, doi: 10.1172/JCl114460.

21. Yýldýz A, Tuncez A, Grbovic E, Polat N, Yuksel M, Aydin M, et al. The association between neutrophil/lymphocyte ratio and functional capacity in patients with idiopathic dilated cardiomyopathy. J Am Coll Cardiol 2013; 62: C101.

22. McGill HC Jr, Anselmo VC, Buchanan JM, Sheridan PJ. The heart is a target organ for androgen. Science 1980; 207: 775-777, doi: 10.1126/science.6766222.

23. Travis A, Amsterdam A, Belanger C, Grosschedl R. LEF-1, a gene encoding a lymphoid-specific protein with an HMG domain, regulates T-cell receptor alpha enhancer function [corrected]. Genes Dev 1991; 5: 880-894, doi: 10.1101/gad. 5.5.880.

24. Swope D, Li J, Radice GL. Beyond cell adhesion: the role of armadillo proteins in the heart. Cell Signal 2013; 25: 93-100, doi: 10.1016/j.cellsig.2012.09.025.

25. Miller JR, Moon RT. Signal transduction through beta-catenin and specification of cell fate during embryogenesis. Genes Dev 1996; 10: 2527-2539, doi: 10.1101/gad.10.20.2527.
26. Bonventre JV, Zuk A. Ischemic acute renal failure: an inflammatory disease? Kidney Int 2004; 66: 480-485, doi: 10.1111/j.1523-1755.2004.761_2.x.

27. Rao A, Luo C, Hogan PG. Transcription factors of the NFAT family: regulation and function. Annu Rev Immunol 1997; 15: 707-747, doi: 10.1146/annurev.immunol.15.1.707.

28. Oka T, Xu J, Molkentin JD. Re-employment of developmental transcription factors in adult heart disease. Semin Cell Dev Biol 2007; 18: 117-131, doi: 10.1016/j.semcdb. 2006.11.012.

29. Diedrichs $\mathrm{H}$, Chi M, Boelck B, Mehlhorn U, Schwinger RH. Increased regulatory activity of the calcineurin/NFAT pathway in human heart failure. Eur J Heart Fail 2004; 6: 3-9, doi: 10.1016/j.ejheart.2003.07.007.

30. Bukowska A, Lendeckel U, Hirte D, Wolke C, Striggow F, Rohnert $P$, et al. Activation of the calcineurin signaling pathway induces atrial hypertrophy during atrial fibrillation. Cell Mol Life Sci 2006; 63: 333-342, doi: 10.1007/s00018005-5353-3.

31. Ebner N, Steinbeck L, Doehner W, Anker SD, von Haehling $\mathrm{S}$. Highlights from the 7th Cachexia Conference: muscle wasting pathophysiological detection and novel treatment strategies. J Cachexia Sarcopenia Muscle 2014; 5: 27-34, doi: 10.1007/s13539-014-0136-z.

32. Xu Q, Ji YS, Schmedtje JF Jr. Sp1 increases expression of cyclooxygenase-2 in hypoxic vascular endothelium. Implications for the mechanisms of aortic aneurysm and heart failure. J Biol Chem 2000; 275: 24583-24589, doi: 10.1074/ jbc.M003894200.

33. Latronico MV, Condorelli G. microRNAs in hypertrophy and heart failure. Exp Biol Med 2011; 236: 125-131, doi: 10.1258/ebm.2010.010269.

34. Gladka MM, da Costa Martins PA, De Windt LJ. Small changes can make a big difference - microRNA regulation of cardiac hypertrophy. J Mol Cell Cardiol 2012; 52: 74-82, doi: 10.1016/j.yjmcc.2011.09.015.

35. Piubelli C, Meraviglia V, Pompilio G, D'Alessandra Y, Colombo GI, Rossini A. microRNAs and cardiac cell fate. Cells 2014; 3: 802-823, doi: 10.3390/cells3030802.

36. Su J, Zhang A, Shi Z, Ma F, Pu P, Wang T, et al. MicroRNA200 a suppresses the Wnt/beta-catenin signaling pathway by interacting with beta-catenin. Int $J$ Oncol 2012; 40: 1162-1170.

37. Ramakrishna S, Kim IM, Petrovic V, Malin D, Wang IC, Kalin TV, et al. Myocardium defects and ventricular hypoplasia in mice homozygous null for the Forkhead Box M1 transcription factor. Dev Dyn 2007; 236: 1000-1013, doi: 10.1002/ dvdy.21113.

38. De Boer I. The effect of prednisolone and isoflupredone on airway hypersensitivity and hyperreactivity in horses with inflammatory airway disease. 2010. Doctoral thesis, Faculty of Veterinary Medicine, Utrecht University, The Netherlands; http://dspace.library.uu.nl/handle/1874/40308.

39. Wagner SA, Apley MD. Pharmacodynamics of isoflupredone acetate in an endotoxin-induced mastitis model. J Dairy Sci 2003; 86: 792-798, doi: 10.3168/jds.S0022-0302(03)73661-3.

40. Brans JW, Lindeboom R, Snoek JW, Zwarts MJ, van Weerden TW, Brunt ER, et al. Botulinum toxin versus trihexyphenidyl in cervical dystonia: a prospective, randomized, double-blind controlled trial. Neurology 1996; 46: 1066-1072, doi: 10.1212/ WNL.46.4.1066. 